\title{
Effect of Knee-to-Chest Position on Primary Dysmenorrhea in School Going Girls
}

\author{
Authors \\ Darpita Patel $^{1^{*}}$, Dr Anuradha Sutar (PT $)^{2}$, Dr Snehal Ghodey (PT) ${ }^{3}$ \\ ${ }^{1}$ BPTH intern MAEER'S Physiotherapy College, Talegaon Dabhade \\ ${ }^{2}$ Associate Professor MAEER'S Physiotherapy College, Talegaon Dabhade \\ ${ }^{3}$ Principal, MAEER'S Physiotherapy College, Talegaon Dabhade \\ *Corresponding Author \\ Darpita Patel \\ Email:darpita796@gmail.com
}

\begin{abstract}
Dysmenorrhea is a painful symptom that accompanies the menstrual cycle. Primary dysmenorrhea occurs when there is no identifiable pelvic disease and tends to occur within 12 months of menarche.

Aim: To study effect of knee to chest position on primary dysmenorrhea in school going girls.

Subjects and Method: 15 subjects were selected in the age group of 12-15 years with primary dysmenorrhea on the basis of inclusion \& exclusion criteria, which received alternate knee to chest position in supine lying, duration for $1^{\text {st }}-4^{\text {th }}$ week $20 \mathrm{sec}$ hold 10 reps progression \& $5^{\text {th }}-8^{\text {th }}$ week 30 sec 20 reps (3 days per week). Pre assessments of subjects were done on first day of first menstrual cycle and post assessments were done on first day of third menstrual cycle using NPRS, VMSD, MMDQS.

Result: There was extremely significant difference in pre mean \& post mean of NPRS, VMSD, MMDQS.

Conclusion: Knee to chest position is effective in reducing primary dysmenorrhea after 8 weeks of intervention.

Keywords: Primary dysmenorrhea, Knee to chest, School going girls.

\section{Introduction}

Dysmenorrhea can be defined as painful menstruation of sufficient magnitude that affects day to day activities ${ }^{1}$.It is characterized by cramp like pain in lower part of the abdomen which may radiate to thighs \& low back occurring before and/or during menstruation ${ }^{10}$. The problems such as irregular menstruation, excessive bleeding, and dysmenorrhea are commonly seen. Dysmenorrhea is the commonest symptom experienced by large number of adolescent girls. ${ }^{(2)}$ It is most common gynaecological complaint in adolescents\& young

women occurring in $60 \%$ to $93 \%$ of school girls \&young women ${ }^{(7)}$. This disease affects $60 \%$ of females, with systemic symptoms like headache (60\%), low back pain, nausea \& vomiting $(80 \%)$, diarrhoea $(50 \%)$, irritability $(30 \%)$ among others ${ }^{(9)}$. Dysmenorrhea can be classified into two subtypes. Primary dysmenorrhoea (PD) is one where there is no identifiable pelvic pathology \& tends to occur within 12 months of menarche. ${ }^{6}$ Women with PD experience sharp, intermittent spasms of pain usually in the suprapubic area. Pain may radiate to the lower back and up to thighs ${ }^{3}$. PD is one of the important causes of school absenteeism amongst
\end{abstract}


adolescent girls, because it affects their academic performance, and other school activities. ${ }^{14}$ Pain usually develops few hours before start of menstruation \& lasts for 24-48 hours \& also peaks as the flow becomes heaviest during the first day to two of the cycle ${ }^{(9)}$

Secondary dysmenorrhoea can occur many years after menarche \&it is associated pain occurring in the presence of pelvic pathology. For example, Endometriosis chronic pelvic infection ${ }^{1}$ Premenstrual syndrome is the collection of emotional symptoms, with or without physical symptoms, related to women's menstrual cycle. The pain associated with PMS is generally related to breast tenderness \& abdominal bloating rather than a lower abdominal cramping pain. PMS symptoms begin before the menstrual cycle and resolve shortly after menstrual flow begins. ${ }^{1}$

\section{Pathophysiology}

The etiology of PD is not precisely understood, but most symptoms of PD can be explained by the action of uterine PGs particularly PGF $2_{\text {alfa. }}$ The disintegrating endometrial cells release $\mathrm{PGF} 2_{\text {alfa }}$ as menstruation begins. PGF $2_{\text {alfa }}$ stimulates myometrial contractions, ischemia and sensitization of nerve endings. Falling progesterone level during the luteal phase brings about these elevations, specifically of

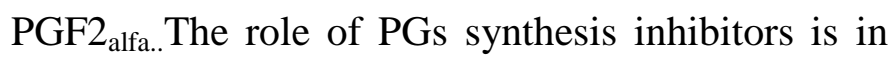
reducing painful symptoms accompanying menstrual discharge. The intensity of the menstrual cramps \& associated symptoms of dysmenorrhea are directly proportional to the amount of PGF2 $2_{\text {alfa }}$ released $^{4,6}$. The levels of PGF $2 \alpha$ are especially high during the first two days of menstruation in women with severe PD. Vasopressin \& leukotriene concentrations have also been found to be higher in women with severe pains than in women who experience mild or no pain ${ }^{3}$. The posterior pituitary hormone vasopressin may be involved in myometrial hypersensitivity, reduces uterine blood flow, and pain in PD. Thus vasopressin role in the endometrium may be related to PGs synthesis and release $^{3}$. Prevalence of dysmenorrhea is $70.2 \%$ in school students and school absenteeism is also more. ${ }^{(15)}$. Several approaches have been proposed for dysmenorrhea, including NSAIDs, oral contraceptives, vitamins, to colytic agents. These drugs have their own side effects and hence women should avoid consuming drugs to prevent unfavourable outcomes and dependence ${ }^{16,17}$. An intervention to relieve pain, apart from drugs, is knee-to-chest position. Improvement is due to increase in the blood flow \& metabolism of uterus during exercise which may be effective in the reduction of dysmenorrhoeal symptoms ${ }^{11}$.

\section{Methodology}

The following is an experimental study carried out by purposive sampling. The study was conducted on students of school going between age group of 1215 years. Subjects were selected based on regular menstrual cycle, NPRS $5 \&$ above. Subjects with secondary dysmenorrhea, premenstrual syndrome, irregular menstrual cycle \& other gynaecological disorder like PCOD were excluded.

\section{Procedure}

Subject's informed consent was taken. Subjects who were selected on the basis of inclusion \& exclusion criteria were given intervention for 8 weeks (3 days per week). Intervention was started on 1 st day of first menstrual cycle, for $1^{\text {st }}-4^{\text {th }}$ week duration was $20 \mathrm{sec}$ hold 10 repetition $\&$ from $5^{\text {th }}-8^{\text {th }}$ week duration was $30 \mathrm{sec}$ hold 20 repetition. Pre assessments were taken on $1^{\text {st }}$ day of first menstrual cycle $\&$ post assessments were taken on $3^{\text {rd }}$ day third menstrual cycle using outcome measures. Outcome measures used were Numerical Pain Rating Scale, Verbal Multidimensional Scoring for Dysmenorrhea, MOOS Menstrual Distress Questionnaire.

\section{Statistical Analysis}

All the statistical analysis was analysed using software 'Graph Pad In Stat v 3.06. Wilcoxon signed rank test was used to ascertain the significance of difference between mean values of two continuous variables. Confidence interval was kept at $95 \%$. 


\section{Results}

There is extremely significant difference in pre \& post mean of NPRS, VMSD score, MMDQS score with $\mathrm{p}$ value $<0.05$.

Table 1:Numerical pain rating scale

\begin{tabular}{|l|c|c|c|}
\hline Pre Mean & Post Mean & Mean Difference & P Value \\
\hline $6.26 \pm 1.03$ & $3.26 \pm 0.88$ & $3.0 \pm 0.92$ & 0.0001 \\
\hline
\end{tabular}

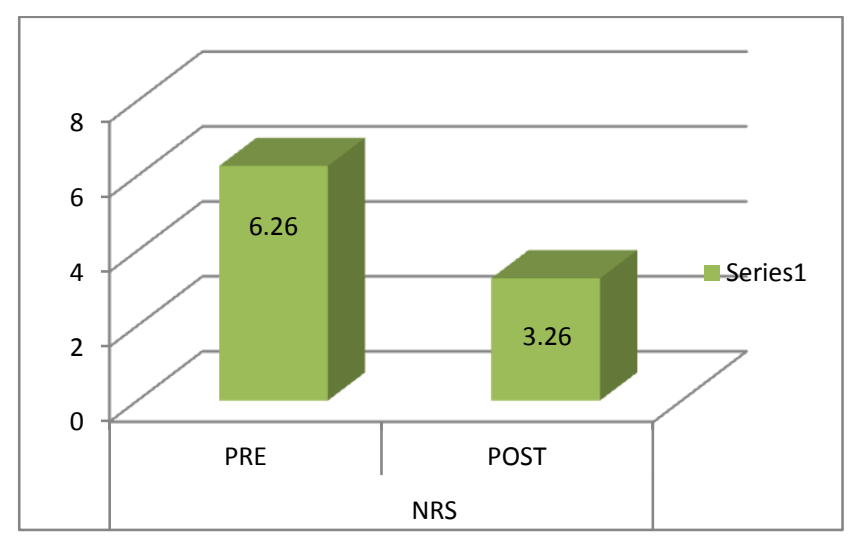

Table 2: Verbal Multidimensional Scoring For Dysmenorrhea.

\begin{tabular}{|l|c|c|c|}
\hline Pre Mean & Post Mean & Mean Difference & P Value \\
\hline $1.66 \pm 0.72$ & $0.66 \pm 0.61$ & $1.0 \pm 0.37$ & 0.0001 \\
\hline
\end{tabular}

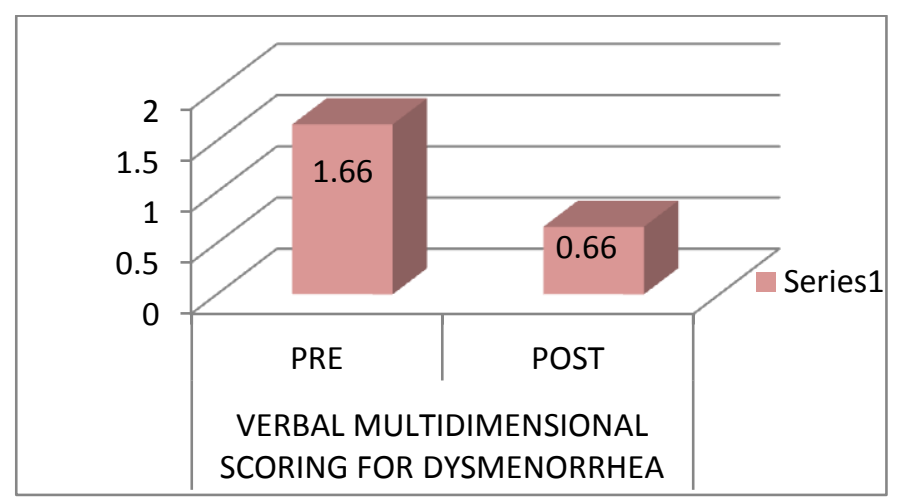

Table 3: MOOS Menstrual Distress Questionnaire

\begin{tabular}{|l|c|c|c|}
\hline Pre Mean & Post Mean & $\begin{array}{c}\text { Mean } \\
\text { Difference }\end{array}$ & P Value \\
\hline $34.86 \pm 11.41$ & $31.13 \pm 11.16$ & $3.73 \pm 0.59$ & 0.0001 \\
\hline
\end{tabular}

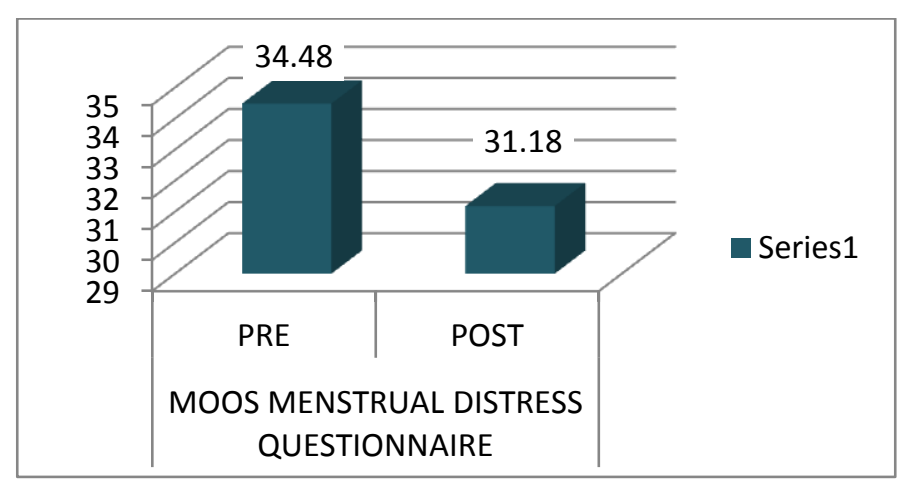

\section{Discussion}

The present study aimed at finding out the effectiveness of knee to chest position on primary dysmenorrhea in school going girls. 15 students in the age group of 12-15 years with PD were selected $\&$ there were 3 drop outs. The results of the present study showed that there was significant difference in pain intensity, VMSD score \& MMDQ. The mean baseline value for pain pre intervention was $6.26 \pm 1.03$. After intervention the mean value for pain was $3.26 \pm 0.88$ with $\mathrm{p}$ value $<0.05$ which suggests that knee to chest position may help in reducing intensity of dysmenorrhea. The pain during menstrual cycleis due to increase in prostaglandins which are synthesis inhibitors which helps in reducing painful symptoms accompanying menstrual discharge. Increase in prostaglandin levels results in uterine contraction \& vasoconstriction and thus causes ischemia to the uterus. Due to fall in progesterone level during luteal phase PGF2 $\alpha$ and PGE2 are elevated. ${ }^{3}$ Thus Knee to chest position reduces dysmenorrhea by increasing blood flow \& also acts on the lining of the uterus \& increases level of circulating endorphins which in turn raise pain threshold.

The increase in pain threshold is due to adjustment of endogenous pain control mechanism. During physical activity body starts to secrete more neurotransmitters such as nor epinephrine, serotonin, encephalin, and dopamine, which acts to inhibit \& control pain. The result of present studies are also consistent with the findings of Abbaspour $\mathrm{Z}$ who concluded that women who exercised at least once per week show significant improvements in reduction of pain. ${ }^{4}$

Eight domains of MMDQ (pain, concentration, behavioural changes, autonomic reaction, water retention, negative effect, arousal, control) showed significant improvement after eight weeks of intervention. The obtained results of my study are agreed with those of Aganoff and Boyle (1994) which concluded that female with primary dysmenorrhea who exercise regularly have significant effect on negative mood state, behaviour change, impaired concentration, and pain. ${ }^{19}$ 
The findings of the above study are in accordance with study done by Arati Mahishale, Dinika Mascarenhas, Shobhana Patted. In that study knee to chest position along with hot moist pack reduces the symptoms of primary dysmenorrhea. ${ }^{2}$

The mean baseline value for VMSD before intervention was $1.66 \pm 0.72$ and post intervention was $0.66 \pm 0.61$ with mean difference $1.0 \pm 0.37$,there were changes in pre and post grade for dysmenorrhea reducing from grade 2 to grade 1 , grade 3 to grade 2 suggesting that knee to chest helps in reducing severity of dysmenorrhea by increasing blood flow to the uterus which reduces ischemia of the uterus. Yuvarani. G, Kamatch. K, Tharani. G, Muthulakshmi. K and Sweetly R also studied effectiveness of knee to chest position along with IFT which concluded that knee to chest position is effective in reducing dysmenorrhea. ${ }^{18}$

Kristina S Gamit in their study concluded that stretching exercise are effective in reducing pain in young females with primary dysmenorrhea. Exercise increases blood flow to the pelvic organs thereby causing wash out of the waste products \& blood clots which are formed in menstruation, which helps in decreasing pain and discomfort during menstruation. The result obtained from knee to chest position are similar to the most study results, which would improve pelvic blood flow, decreasing muscle stresses, providing stretching of all involved structure with consequent pain relief. ${ }^{3}$ Billing in 1943 proposed that women with PD had contracted ligamentous bands in the abdomen \& he subsequently developed a series of stretching exercises for which he claimed a high rate of symptom relief ${ }^{4,5}$. The results are also supported by those reported by Hood and Dincher (1992) who suggested a program of exercise for dysmenorrhea patients 2-3 times a day before the period which includes; supine lying position with head and legs raised simultaneously with arms kept at sides and legs straight, deep knees bent with back kept straight and walking on hands and feet. They reported that exercise reduces pain during menstruation by relaxing abdominal muscles and relieves pressure on the nerve centre, pelvic organs and decreases pelvic congestion. ${ }^{21}$

\section{Conclusion \& Clinical Implication}

The present study concluded that knee to chest position is effective in reducing the menstrual pain $\&$ discomforts of primary dysmenorrhea. Our findings showed that exercise can help in reducing pain and improve physical symptoms which overall improves quality of life of girls with primary dysmenorrhea by reducing school absenteeism \& improve school performance. Dysmenorrhea as it is a significant problem for girls, that causes absence from school, college \& work, we can recommend regular exercise as a helpful means in this age group. Knee to chest position can be incorporated in clinical practice as it is easy to perform $\&$ it is cost effective. This exercise can also be performed at home $\&$ can also be given in community of any age group. Thus knee to chest position can be used as an adjunct to improve dysmenorrhea rather than using NSAIDs, oral contraceptives etc.

\section{Scope}

Study should be concluded with larger sample size and duration. Study also aims at treating females with dysmenorrhea due to secondary conditions.

\section{Limitation}

Sample size was small.

There were 3 drop outs from the project because of academics.

\section{Acknowledgment}

The authors would like to thank the support of the staff of the MAEER's Physiotherapy College, Talegaon Dabhade and also like to thank subjects for their willingness to participate in the study.

\section{References}

1. D.C Dutta Textbook of Gynecology $6^{\text {th }}$ Edition, ISNBN-7381-174

2. Arati Mahishale, Dinika Mascarenhas, Shobhana Patted. Effect of knee chest postion in primary dysmenorrhea (2014) 
3. Anuradha Sutar, Sayli Paldhikar, Nigar Shikalgar, Snehal Ghodey. Effect of aerobic exercise on primary dysmenorrhea in college students. Volume 5, Issue 5 Ver. V (Sep. Oct. 2016), PP 20-24.

4. Abbaspour Z. MSc, Rostami M. MSc, Najjar Sh. MSc seen.The Effect of Exercise on Primary Dysmenorrhea. Iran J Res Health Sci, Vol 6, No 1, pp. 26-31, 2006.

5. Billig 1943 Billig HE Jr. Dysmenorrhoea, the result of a postural defect. Symptom changes across the menstrual cycle in competitive sportswomen, exercisers and sedentary women. British Journal of Clinical Psychology 34, 1995, 447-60.

6. DawoodAdvances in Primary Dysmenorrhea Williams and Wilkins; 1981.p. 2052VOL.108, NO. 2, AUGUST 2006.

7. Derseh BT, Afessa N, Temesgen M, Semayat YW, Kassaye M: Prevalence of Dysmenorrhea and its Effects on School Performance: A Cross-sectional Study. Jet al. (2017):

8. Izzo A, Labrila D. Dysmenorrhea and sports activity in adolescents. Clin Expobstet Gynecol 1991;18:109-16. 10.

9. Kiranmayi P, Ponmathi P, Sivakumar VPR (2016):Comparison of Aerobic versus Stretching Exercise Programmes on Pain and Menstrual Symptoms in Subjects with Primary Dysmenorrhea. J Women's Health Care 5:327. doi:10.4172/21670420.1000327.

10. Kristina S Gamit, Megha S Sheth, Neeta J Vyas Effect of stretching exercise on primary dysmenorrhea in adult girl(2014).

11. Ozlem Onur, MD Impact of home-based exercise on quality of life of women with primary dysmenorrhea SAJOG • January 2012, Vol. 18, No.1

12. Noorbakhsh M, Alijani , Kohandel M, Mehdizadeh Toorzani Z, Mirfaizi M, Hojat $\mathrm{S}$. The effect of physical activity on primary dysmenorrheal of female university students.(2012)
13. MOOS

MENSTRUAL

DISTRESS QUESTIONNAIRE by Rudolf Moos.(2010).

14. Sandeepkaur, Prabhnoorkaur, sarvananshanmugan, manpreetkaurkang: To compare the effect of stretching and core strengthening exercises on primary dysmenorrhea in young female volume 13,issue 6 ver.v,pp 22-26 (2014)

15. Shabnam Omidva, Fatemeh Bakouei, Khyrunnisa Begum: Primary dysmenorrhea \& Menstrual Symptoms in Indian female students :Prevalance, Impact, Management (2015).

16. Witt CL, Reinhold T, Brinkhaus B, Roll S, Jena S, Willich SN: Acupuncture in patients with dysmenorrhea: a randomized study on clinical effectiveness and cost-effectiveness in usual care. Am J Obstet Gynecol. 198(2), 166.e1-e8.(2018)

17. Yeh ML, Hung YL et al: Auricular acupressure for pain relief in adolescents with dysmenorrhea: a placebo-controlled study. J Altern Complement Med. 19(4), 313-8.(2013)

18. Yuvarani. G, Kamatch. K, Tharani. G, Muthulakshmi. K and Sweetly R."Effect of knee chest position with interferential therapy and interferential therapy alone in reducing pain on female students with primary dysmenorrhea". (2017)

19. Aganoff, J. and Boyle, G.: "Aerobic exercise, mood states and menstrual cycle symptoms", J. Psychosom. Res., 38(3): 183-192, 1994.

20. Hood, G. and Dincher, J.: "Total patient care in dysmenorrhoea", $8^{\text {th }}$ ed., Foundations and practice of Adult Health Nursing, Mosby, pp: 1-2, 1992. 